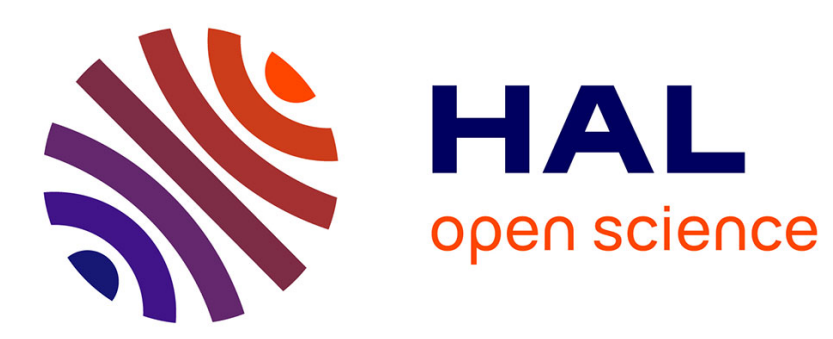

\title{
Bathymay: la structure sous-marine de Mayotte révélée par l'imagerie multifaisceaux
}

Jean-Christophe Audru, Pol Guennoc, Isabelle Thinon, Olivier Abellard

\section{To cite this version:}

Jean-Christophe Audru, Pol Guennoc, Isabelle Thinon, Olivier Abellard. Bathymay: la structure sous-marine de Mayotte révélée par l'imagerie multifaisceaux. Comptes Rendus Géoscience, 2006, 338, pp.1240-1249. 10.1016/j.crte.2006.07.010 . hal-00870100

\section{HAL Id: hal-00870100 https: / hal-brgm.archives-ouvertes.fr/hal-00870100}

Submitted on 28 Oct 2013

HAL is a multi-disciplinary open access archive for the deposit and dissemination of scientific research documents, whether they are published or not. The documents may come from teaching and research institutions in France or abroad, or from public or private research centers.
L'archive ouverte pluridisciplinaire HAL, est destinée au dépôt et à la diffusion de documents scientifiques de niveau recherche, publiés ou non, émanant des établissements d'enseignement et de recherche français ou étrangers, des laboratoires publics ou privés. 
Océanographie / Oceanography

Bathymay : la structure sous-marine de Mayotte révélée par l'imagerie multifaisceaux Bathymay : underwater structure of Mayotte island revealed by multibeam bathymetry Jean-Christophe Audru ${ }^{1}$, Pol Guennoc ${ }^{2}$, Isabelle Thinon ${ }^{2}$ et Olivier Abellard ${ }^{3}$ 1 : BRGM, antenne de Mayotte, BP 1398, 97600 Mamoudzou 2 : BRGM, CDG/MA, BP 6009, 45060 Orléans cedex 02 3 : DAF, Service des Pêches, BP 103, 97600 Mamoudzou

\author{
Correspondance : \\ Jean-Christophe Audru \\ BRGM, antenne de Mayotte, BP 1398, 97600 Mamoudzou \\ Courriel : jc.audru@brgm.fr \\ Tél. 0269612813 \\ Fax. 0269612815
}


Océanographie / Oceanography

\section{Résumé}

La campagne BATHYMAY, effectuée en 2004 à bord du N/O Marion Dufresne 2, a permis de réaliser le premier levé bathymétrique complet de la pente insulaire de Mayotte.

L'analyse préliminaire de la morphologie révèle l'existence de nombreux canyons, de vastes déstabilisations de flancs et d'édifices volcaniques sous-marins. Des falaises abruptes, dont certaines semblent guidées par des failles, limitent de vastes plateaux sous-marins. La campagne Bathymay a mis en évidence deux mécanismes de déstabilisation du volcan de Mayotte : la déstabilisation lente, issue de l'effondrement gravitaire de l'édifice pendant le stade bouclier, puis la déstabilisation catastrophique des flancs du volcan au stade postbouclier.

\section{Abstract}

The BATHYMAY marine survey was carried out in January 2004 around the French volcanic island of Mayotte, onboard the research vessel Marion Dufresne 2. Multibeam bathymetry revealed for the first time the morphology of the whole outer slope. Preliminary interpretations show broad canyons which deeply incise the slope, numerous volcanoes and huge landslides. Large submarine plateaus are also highlighted, bounded by steep cliffs and active normal faults. A model for Mayotte is suggested, in which eruptive activity, sometimes influenced by normal faulting, was concentrated along a $\mathrm{N} 140^{\circ}$ regional line (the Comoros archipelago axis), with landslide activity concentrated on the NE and SW flanks of this line.

Mots-clés : bathymétrie, imagerie, volcans, risques, Mayotte, Comores

Key-words : bathymetry, imagery, volcanoes, hazards, Mayotte, Comoros 
Océanographie / Oceanography

\section{Abridged English version}

\section{Introduction}

Mayotte is a French Overseas Territory of the Comoros archipelago (Figure 1a). It includes two main islands, Grande-Terre and Petite-Terre (total area about $370 \mathrm{~km}^{2}$ and population around 165000 inhabitants). The two islands are surrounded by a large lagoon $\left(1100 \mathrm{~km}^{2}\right)$, which is almost completely delimited on $150 \mathrm{~km}$ by a substantial barrier reef cut channels (Figure 1b).

The volcanic Comoros archipelago is derived from the drift of the Somali plate on top of a hot spot plume $[12,26]$. Evidence of the hot spot volcanic chain extends from the Glorioso atoll (250 km to the NE of Mayotte) to the Grande-Comore active volcano (Karthala, $2263 \mathrm{~m}$ ) 250 $\mathrm{km}$ to the NW. Mayotte probably resulted from the evolution of two shield-volcanoes which were submarine from 15 to $10 \mathrm{My}$ and subaerial post-10 My. The main units that outcrop on land (Figure 1b) are basaltic, phonolitic or trachytic lavas, pyroclastic deposits [27] and large thickness of argillaceous weathered rocks. Geomorphological observations on land and offshore [1] suggest the existence of large normal faults (N045 ${ }^{\circ}$ and $\left.\mathrm{N} 160-180^{\circ}\right)$. Other directions of fractures are inferred to result from post-eruptive extension (tectonic or gravity driven). Volcanism continued until the Quaternary around Petite-Terre with ashes dated at approximately 7000 years BP [ 32 ]. The reef started to build about $1.5 \mathrm{My}$ ago [29, 30]. The lagoon and the reef were emergent during the Last Glacial Maximum (LGM) 26000 years ago, forming channels and karst systems currently at $150 \mathrm{~m}$ depths [10]. The lagoon was flooded 11600 years ago [32].

Since 2001, the BRGM (the French geological survey) and the Department Council have been evaluating and mapping the onshore natural hazards of Mayotte. The structure and the sedimentology of the lagoon are already documented [29, 30, 32]. A detailed bathymetry of the outer slope, reported in this paper, was necessary to complement these onshore and shallow marine studies. 


\section{Survey conditions and observed features}

Detailed multibeam bathymetry data were acquired for 3.5 days around Mayotte island, onboard the R/V Marion-Dufresne 2. The whole outer slope was mapped during the survey, encompassing approximately 8750 sq. km (i.e. 2550 sq. nmi). We present here the morphological analysis (Figure 3) of a digital elevation model (DEM) obtained through $50 \mathrm{~m} \times 50 \mathrm{~m}$ gridding (Figure 2) of the multibeam data.

On the oceanic floor, the base of Mayotte island exceeds $110 \mathrm{~km}$ in diameter, $10500 \mathrm{~km}^{2} \mathrm{in}$ area and $4400 \mathrm{~m}$ in altitude, which implies that only $4 \%$ of Mayotte is emergent (Figure $2 \mathrm{~b}$ ). Depths extend from $-44 \mathrm{~m}$ to $-3767 \mathrm{~m}$ below sea level. In the north and east, the reef rim forms continuous slopes down to $1000 \mathrm{~m}$ depths, whereas to the south and west, the reef connects to the abyssal plain through two submarine plateaus.

Further plateaus, canyons, landslides, cliffs and volcanoes have been located on the outer slope (Figures 2 and 3). .

To the south and west, the bathymetry highlights two submarine plateaus slightly tilted westwards $\left(1.5\right.$ to $\left.2^{\circ}\right)$. The southwestern plateau $(P 1)$ is deeper $(750 \mathrm{~m})$ and larger $\left(240 \mathrm{~km}^{2}\right)$ than the northwestern one (P2) which covers $100 \mathrm{~km}^{2}$ at $600 \mathrm{~m}$ depths. Their surfaces are cut by channels that may record subaerial erosion. Using an average rate of subsidence of $19 \mathrm{~cm}$ per 1000 years [4], the north-western plateau would have been sub-aerial 2,6 My ago and the south-western plateau 3,9 My ago.

The circular depressions observed on $P 1$ may correspond to craters or lakes such as those that formed in the lagoon of New Caledonia when it was emergent [5].

The canyon network covers the whole outer slope of Mayotte. Their cumulative length exceeds $1700 \mathrm{~km}$ and the troughs can reach $50 \mathrm{~km}$ in length, 2 to $3 \mathrm{~km}$ in width and $350 \mathrm{~m}$ in vertical incision. Most of the canyons form a regular radial network, though some abrupt changes in direction (of $15^{\circ}$ with $30^{\circ}$ ) are observed, probably due to faults $(F 2)$ or other structural features $(C 1)$. Some canyons are located immediately downstream of channels of the lagoon (C2), where they form the prolongation of the lagoon drainage network when it was still emergent $[10,32]$. There appear to be two types of deeper water canyons: deep 
valleys of a hundred meters to less than $1 \mathrm{~km}$ in width (C3) that lack well-developed headwater tributary systems and broader and less incised canyons (C4) with substantial tributary canyons and channels. Variations in acoustic reflectivity (still being analysed) suggest that channels are characterized by the absence of fill (suggesting erosional currents) or by the existence of coarse deposits. All these canyons would result from the initial gravity collapse of the shield-volcanoes, as observed in experiments [22], together with erosion due to turbidity currents during the LGM [30].

Landslides have occurred mainly on the outer slope in the NE (S1) and SW (S2) of the island. Their longitudinal extension reaches $35 \mathrm{~km}$ and their area exceeds $1800 \mathrm{~km}^{2}$. Smaller landslides are inferred in the SE (S3) and west (S4). The surfaces of these debris avalanches are similar to "hummocky surfaces" observed offshore Hawaii [18] or off French Polynesia [6]. The erosion by turbidity currents of these soft deposits may generate canyons [22], which are obviously more recent than those of non slipped zones. The S3 slip at the foot of a cliff, as well as the structural position of this cliff close to F2 inferred normal faults (Figure $1 b$ ), suggest that the $F 1$ cliff marks an active fault scarp. The concave morphology (S3) observed in the southeast of Mayotte (Figure 3) is interpreted as the trace of a gravity collapse structure involving the whole slope and reef. The two volcanoes, still present in the scar are thought to be responsible for the landslide. Such intrusions and collapses are observed in other volcanoes like Saint-Helens [8] or Hawaii [11]. The implication of the reef in collapse indicates the structure is less than 1,5 My old.

Large underwater landslides are destructive phenomena, commonly observed around other volcanoes as Hawaii [18], Canaries [1], French Polynesia [6] or La Reunion for example $[15,21]$. Larger ones may also generate earthquakes or tsunamis. At this stage of the study, we note that 1936 to 2001 epicentres are located close to the front of the landslides (Figure 3). Also, Mayotte oral history reports [13] some past tsunamis, but no field evidence has been reported yet. 
More than 200 cones or crater-like structures, interpreted as volcanoes, have been noted on the outer slope (Figure 3). They exceed 2 to $3 \mathrm{~km}$ in diameter and their distribution is particularly high in the northwest (V1), starting from $2000 \mathrm{~m}$ depth and in the east (V2) and from $600 \mathrm{~m}$ depths in the SE (V3). Some conical shapes rise up to $500 \mathrm{~m}$ above the oceanic floor, which is comparable with the dimensions of volcanoes observed on land. The widening at the top of craters is interpreted as the collapse of the roof intrusion, due to high pressure at these depths.

\section{Interpretation}

Landslides areas are preferentially located in the northeast and southwest, whereas volcanic swarms are located in the northwest and southeast. This "cross" distribution, as well as the general morphology of the outer slope, help to explain the evolution of the island.

The southwestern volcanic unit (V4) corresponds to the Mayotte southern shield volcano, dated onland as 10 My [7, 27]. It outcrops in a large $\mathrm{N} 140^{\circ}$ caldera [27]. On land, the volcanic series comprises mainly Mio-Pliocene basaltic lava and Pliocene phonolite necks [7]. Its very mature topography makes it the oldest geological feature of Mayotte.

The northeastern unit (V5) corresponds to the northern shield-volcano, where onland dating gives 4 My ages [7, 27]. The northwestern region of Mayotte (V6) is characterized by a narrow outer slope and is bounded to the east by a probable fault scarp ( $F 1$ to F2). Pliocene basalts and phonolites [7, 27] are found onland. Mayotte island thus comprises three main volcanoes (V4, V5 and V6) on which late smaller volcanoes erupted (V1, V2 and V3).

The southeastern part of the island is characterized by numerous volcanic cones. On land, late Quaternary pyroclastic maars and cones have intruded through the reef itself $[7,27,32]$. This area represents the most recent volcanic field of Mayotte.

The decompression generated by the normal faults to the northwest and by gravity collapse to the southeast probably influenced the concentration of the recent eruptive activity in these sectors; such features are observed in the Canaries islands and along the Kilaua volcano in Hawaii $[1,19]$. 


\section{Conclusions}

The structures revealed by detailed multibeam bathymetry show a complex evolution for Mayotte. The island comprises three main Miocene to Pliocene volcanoes and three Holocene volcanoes. As in many volcanic islands, canyons reflect the strength of erosion and transfer of detritic material during the shield-stage. The post-shield stage seems mainly controlled by the fast destabilization of the volcano and includes huge landslides and normal faulting. The latter may have controlled the distribution of the late eruptive activity.

A model for Mayotte is suggested in which the eruptive activity was concentrated along a $\mathrm{N} 140^{\circ}$ regional line (the Comoros axis), with landslides concentrated on the northeastern and southwestern flanks of this line.

\section{Géologie sommaire de Mayotte}

Mayotte est une Collectivité Départementale française de l'archipel des Comores (Figure 1a). Elle comprend deux îles principales, Grande-Terre et Petite-Terre dont la superficie totale est de $370 \mathrm{~km}^{2}$ et la population d'environ 165000 habitants (Insee , 2002). GrandeTerre et Petite-Terre sont entourées du plus vaste lagon fermé de l'océan Indien $\left(1100 \mathrm{~km}^{2}\right)$; ce lagon est presque totalement délimité sur $150 \mathrm{~km}$ par une barrière récifale entrecoupée de quelques passes (Figure 1b).

L'archipel volcanique des Comores provient de la dérive de la plaque somalienne au dessus d'un point chaud $[12,26]$, dont on retrouve la trace depuis l'atoll des Glorieuses à $250 \mathrm{~km}$ au Nord-Est de Mayotte, jusqu'au volcan actif de Grande-Comore à 250 km au Nord-Ouest de Mayotte (Figure 1a). L'analyse géochimique des laves comoriennes suggère qu'elles sont issues d'interactions croissantes entre un panache mantellique et la lithosphère océanique encaissante [7]. Mayotte résulterait de l'évolution de deux volcans boucliers depuis 15 millions d'années (Ma) en domaine sous-marin et depuis $10 \mathrm{Ma}$ en domaine aérien pour le bouclier Sud, et environ $4 \mathrm{Ma}$ pour le bouclier Nord [7, 27]. Les lithologies principales observées sur le terrain (Figure 1b) sont des empilements de coulées de type basaltique, 
phonolitique ou trachytique, des scories basaltiques et des dépôts pyroclastiques à cendres, lapilli et blocs [27]. Les épisodes d'activité volcanique ont été entrecoupés de périodes d'altération supergène (sur basaltes) et/ou hydrothermale (sur phonolites) qui se traduisent, sur le terrain, par des altérites argileuses interstratifiées ou imbriquées dans les coulées.

L'importance de la couverture argileuse rend difficile la compréhension structurale. On peut cependant formuler quelques hypothèses, basées principalement sur des observations

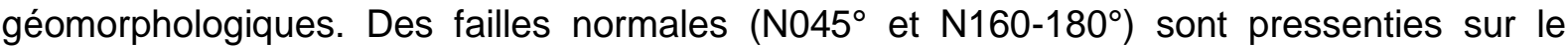
terrain et dans le lagon, en particulier dans le Nord-Ouest où elles limiteraient des blocs basculés [2,3] (Figure 1b). Ces failles réactiveraient des structures fossiles qui ont accompagné l'océanisation jurassique du bassin de Somalie [16]. Des dykes intrusifs dans des altérites mio-pliocènes sont relevés dans le Nord-Ouest de Mayotte avec des orientations récurrentes $\mathrm{N} 140^{\circ}$, correspondant à l'axe de l'archipel des Comores depuis l'édification de Mayotte. D'autres fractures de directions variées sont relevées, elles résulteraient plutôt d'extensions post-éruptives d'origine tectonique ou gravitaire.

L'activité volcanique se poursuit jusqu'au Quaternaire dans le Nord-Est de Mayotte : à terre, des basaltes vacuolaires datés à 150000 ans BP [7] sont nappés de dépôts pyroclastiques à cendres et ponces d'origine phréato-magmatique ; dans le lagon, des carottages ont fourni des cendres datées à environ 7000 ans BP [32]. Au large des reliefs, la barrière récifale commence à s'édifier il y a approximativement 1,5 Ma, avec une accélération de la construction à l'Interglaciaire il y a 125000 ans BP [29, 30]. Le lagon et le récif sont ensuite exondés à la faveur du Dernier Maximum Glaciaire (DMG) de 26000 ans BP, entraînant la création des passes et des systèmes karstiques qui ont été observés sur la pente externe par submersible à -150 m [10]. L'ennoiement progressif du lagon reprend par les passes il y a 11600 ans BP [32].

Le taux moyen de subsidence de l'archipel de Mayotte est évalué à 0,19 mm/an d'après des datations de récifs [4]. Enfin, l'activité sismique modérée enregistrée à Mayotte comporte trois séismes récents $(M=5$ et 5.2 en 1993 et $M=4.1$ en 2001) dont les épicentres sont localisés dans l'Ouest et le Nord de Mayotte (Figure 3). Cette activité est attribuée à la 
migration de la déformation du rift est-africain vers le canal de Mozambique [20] puis vers Madagascar [3, 16, 26].

La structure et la sédimentologie du lagon de Mayotte sont déjà bien documentées [29, 30, 32]. En 2002, la numérisation puis l'interpolation de points de sonde de cartes du SHOM [2 ; 24] ont permis de montrer que la partie Ouest du lagon était en moyenne plus profonde que la partie Est (60 m vs. 30 m). Confirmant les observations de plongeurs, cette cartographie a également révélé l'existence de paléo-vallées profondes (chenaux incisés sur $60 \mathrm{~m}$ de profondeur et $300 \mathrm{~m}$ de largeur) en prolongement des grandes ravines actuelles et à l'amont de certaines passes. L'existence d'anciens lacs (dépressions ovales) fonctionnels pendant les périodes d'émersion du lagon, est également pressentie, comme c'est le cas par exemple dans le lagon de Nouvelle-Calédonie [5].

Par contre, les flancs de l'édifice volcanique à l'extérieur du lagon restaient peu connus. Des profils topographiques et des études sédimentologiques ont été réalisées à partir de campagnes de plongées en submersible entre 1998 et 2003 [10, 30]. La cartographie évoquée ci-dessus avait permis d'identifier, sur la pente externe, des morphologies évoquant des cônes volcaniques, des glissements sous-marins, des falaises et des sillons pouvant représenter des failles ou des canyons [2, 3]. Par ailleurs, à terre, en partenariat avec la Collectivité Départementale, le BRGM menait depuis 2001 un programme d'évaluation et de cartographie des aléas naturels. Une connaissance plus détaillée de la morphologie des zones immergées était donc nécessaire pour compléter cette approche.

\section{Objectifs et déroulement de la campagne Bathymay}

La campagne BATHYMAY avait deux objectifs: 1) la cartographie bathymétrique par sondeur multifaisceaux de la pente insulaire externe au lagon de Mayotte et 2) la morphologie des zones potentielles de ressources halieutiques. II s'agissait en effet, pour la DAF de disposer de levés bathymétriques fins pour le développement de la pêche hauturière à Mayotte. 
Les levés ont été effectués du 7 au 12 janvier 2004 à bord du navire océanographique Marion-Dufresne 2 (Figure 2a). Ils représentent 750 miles de levés en mode mixte (bathymétrie, sondeur à sédiment, gravimétrie et magnétisme) et 230 miles en mode simple (sondeur à sédiment) à la vitesse moyenne de 13 nœuds, Au total, la superficie de la zone cartographiée dépasse $8750 \mathrm{~km}^{2}$. Les données ont été traitées à bord par le SHOM et le BRGM avec le logiciel CARIS-HIPS puis avec le logiciel Caraïbes de l'IFREMER pour l'élimination des artéfacts majeurs. Nous présentons ici les données bathymétriques de la campagne sous la forme d'un Modèle Numérique de Terrain calculé à la maille de 50 mètres (Figure 2a) puis les premières analyses de la morphologie et des structures observées. À partir de ce MNT bathymétrique, une interprétation est proposée en Figure 3.

\section{Les principaux traits morphostructuraux}

La cartographie d'ensemble du relief sous-marin montre que l'embase de l'archipel de Mayotte dépasse $110 \mathrm{~km}$ de diamètre sur le plancher océanique, pour une superficie de $10500 \mathrm{~km}^{2}$ : cela implique que seulement $4 \%$ de la surface de Mayotte sont émergés (Figure 2b). Les profondeurs insonifiées s'étendent de -44 m (relief à l'Est de Petite-Terre) jusqu'à -3767 m (à l'Ouest de Grande-Terre), ce qui confère à Mayotte une altitude d'au moins $4400 \mathrm{~m}$ au-dessus de la plaine abyssale. Les pentes se situent en majorité entre $4^{\circ}$ et $20^{\circ}$ avec des valeurs maximales à $88^{\circ}$ au niveau de falaises situées dans l'Ouest de Mayotte (Figure 2b). Au Nord et à l'Est, la barrière récifale borde des pentes continues jusqu'à $1000 \mathrm{~m}$ de profondeur, alors qu'au Sud et à l'Ouest, la barrière se raccorde à la plaine abyssale par l'intermédiaire de deux plateaux sous-marins. Les deux principales orientations observées dans la morphologie générale de la pente insulaire de Mayotte sont $\mathrm{N} 140^{\circ}$ au Nord et $\mathrm{N} 015^{\circ}$ au Sud. Cette dernière direction ne correspond à aucune structure ou direction observée à terre.

Diverses structures géomorphologiques ont pu être repérées sur la pente insulaire : des plateaux, des falaises, des canyons, des zones « rugueuses » ainsi que de nombreux petits reliefs isolés (Figures 2 et 3 ). 
Sur la partie occidentale du relief insulaire, la bathymétrie met en évidence deux plateaux sous-marins faiblement inclinés vers l'Ouest $\left(1,5\right.$ à $\left.2^{\circ}\right)$. Le plateau Sud-Ouest $(P 1)$ est le plus profond $(750 \mathrm{~m})$ et le plus vaste $\left(240 \mathrm{~km}^{2}\right)$, tandis que celui du Nord-Ouest $(P 2)$ forme une terrasse à $600 \mathrm{~m}$ et ne couvre que $100 \mathrm{~km}^{2}$. Le plateau $P 1$ est incisé par des chenaux et des dépressions circulaires de 300 à 400 m de diamètre (Figure 2a). Chaque plateau est limité à l'aval par des falaises, dont le dénivelé peut atteindre 600 à $700 \mathrm{~m}$; l'arête des falaises est indentée par des canyons, des chenaux étroits ou encore des formes d'incisions subcirculaires. Le plateau Sud-Ouest est limité au Nord par un escarpement linéaire de $5 \mathrm{~km}$ de long et $250 \mathrm{~m}$ de haut $(F 1)$.

Les canyons représentent les morphostructures les plus nombreuses sur l'ensemble de la pente insulaire de Mayotte. La longueur de «drainance » cumulée de ce réseau dépasse $1700 \mathrm{~km}$. Les chenaux d'écoulement peuvent atteindre $50 \mathrm{~km}$ de longueur, 2 à $3 \mathrm{~km}$ de largeur et $350 \mathrm{~m}$ d'incision verticale. Les canyons dessinent un réseau d'ensemble relativement homogène en disposition radiale, suivant généralement les lignes de plus grande pente. Cependant de brusques changements de direction (de $15^{\circ}$ à $30^{\circ}$ ) sont observés dans certains secteurs (C1). L'amont de certains canyons est situé à l'exutoire immédiat des passes du lagon (C2). Les autres canyons évoluent en profondeur selon deux configurations : les uns en vallées individuelles profondes d'une centaine de mètres et de moins de $1 \mathrm{~km}$ de largeur (C3) et les autres, en vallées confluentes dans des canyons collecteurs plus larges et moins incisés (C4). La quasi-totalité des chenaux est caractérisée par une réflectivité acoustique forte, qui peut être expliquée par l'absence de dépôt lié à un gradient élevé et/ou à l'existence de dépôts grossiers.

La bathymétrie (Fig. 2, 3) révèle aussi l'existence de vastes zones à faible relief, non incisées, concentrées sur la pente insulaire au Nord-Est (S1) et au Sud-Ouest (S2) de l'île. Leur extension longitudinale atteint $35 \mathrm{~km}$ et leur superficie dépasse $1800 \mathrm{~km}^{2}$. Des secteurs moins vastes sont également relevés au Sud-Est (S3) et à l'Ouest (S4). Ces reliefs, délimités 
par les canyons, s'élargissent en direction du pied de pente. En amont, des indentations ou des formes sub-circulaires nettes entaillent l'arête des plateaux ou de la pente externe. L'ombrage du MNT souligne la rugosité des zones $S 1$ et $S 2$, due à des irrégularités topographiques linéaires, perpendiculaires à la pente et espacées d'environ 1,5km.

Plus de 200 reliefs coniques de petites dimensions (2 à $3 \mathrm{~km}$ de diamètre ) ont pu être recensés sur la pente insulaire de l'île (Figure 3). Leur densité est particulièrement élevée à partir de $2000 \mathrm{~m}$ de profondeur au Nord-Ouest (V1) et dès $600 \mathrm{~m}$ de profondeur à l'Est (V2) et au Sud-Est (V3). Ces reliefs coniques s'élèvent de 100 à 500 m environ au dessus de la pente insulaire, altitudes comparables aux reliefs volcaniques observés à terre ; ils sont en majorité isolés mais quelque-uns apparaissent coalescents. On note aussi que certains présentent un sommet tronqué. Au pied de l'escarpement sub-circulaire au Sud-Est de l'île (S3), deux cônes de plus de $400 \mathrm{~m}$ de hauteur sont observés à $600 \mathrm{~m}$ de profondeur (V3). On remarque que ces reliefs isolés n'apparaissent pas dans les zones à surface rugueuse décrites précédemment.

\section{Interprétation des structures}

L'analyse de la morphologie de la pente insulaire de Mayotte a permis de mettre en évidence différents types de structures dont nous discutons l'origine.

Les plateaux sous-marins ( $P 1$ et $P 2)$ ont une surface irrégulière : relativement plane dans certains secteurs, elle peut être profondément incisée par quelques chenaux dans d'autres secteurs. II est possible que cette incision soit intervenue alors que les plateaux correspondaient à des zones d'érosion sub-aérienne ou à un platier fossile par exemple, servant de zones de transit de sédiments (by-pass) depuis le paléo-lagon vers les grands fonds. En effet, considérant un taux de subsidence approximatif de $19 \mathrm{~cm}$ par 1000 ans [4], on peut évaluer que le plateau du Nord-Ouest était sub-aérien il y a 2,6 Ma et celui du SudOuest il y a 3,9 Ma. Les dépressions circulaires observées sur P1 pourraient représenter 
des cratères ou bien d'anciens lacs analogues à ceux décrits dans le lagon de NouvelleCalédonie [5].

La présence de la plate-forme $P 1$ s'accorde avec la dissymétrie qui est également observée dans la bathymétrie du lagon [2] (Cf. supra), qui pourrait indiquer une subsidence plus importante, accélérée par la tectonique extensive, de la partie Ouest de l'archipel. La sédimentation récente à la surface de ces plateaux doit être étudiée à partir de carottages réalisés dans le cadre du projet «Cabamay» (Laboratoire Geomar de Kiel) entrepris conjointement avec la campagne Bathymay.

Les canyons sous-marins sont présents sur l'ensemble des flancs de Mayotte depuis la base du récif jusqu'en pied de pente. Ce type d'incision a été observé sur les flancs de divers volcans boucliers, à la Réunion [21] ou aux Canaries [31] par exemple. Des expérimentations analogiques [22] suggèrent que l'effondrement gravitaire initial des volcans boucliers [17], créant des horsts et des grabens « en étoile », est propice au développement des canyons. Par ailleurs, plusieurs canyons sous-marins de la pente de Mayotte sont situés à l'aval immédiat des passes $(C 1)$; ils forment le prolongement profond du réseau hydrographique du lagon lorsqu'il était encore émergé $[10,32]$, à l'instar de ce qui est observé en Nouvelle-Calédonie par exemple [5, 9]. À Mayotte, lors du DMG, les fleuves et leur charge sédimentaire issus des reliefs circulaient dans le lagon alors exondé [30], traversaient les passes puis se connectaient aux têtes des canyons. Sur la pente externe, les sédiments insulaires devaient s'ajouter aux glissements des têtes de canyons (surcharges en tête de talus) puis étaient transportés sous forme d'avalanches vers la plaine abyssale. Le creusement des canyons de Mayotte proviendrait ainsi de l'affaissement gravitaire des volcans boucliers, puis de leur surcreusement par les courants de turbidité à forte charge sédimentaire lors du DMG. La distribution ubiquiste de ces canyons témoigne de l'importance des processus d'érosion et de transferts de matière sur la pente insulaire de Mayotte durant les différents stades de son évolution. Enfin, un canyon semble guidé par un réseau de failles normales présumées (F2) dans le lagon et sur la pente externe. 
Les reliefs « rugueux » ou « fripés » sont interprétés comme le résultat de déstabilisations de flancs ou de glissements sous-marins de grande ampleur (S1 et S2). Ce sont des avalanches de débris dont l'aspect est similaire, par exemple, aux « hummoky surfaces » des glissements observés à Hawaii [18] ou en Polynésie [6]. Les matériaux, issus des bordures des plateaux ou de la pente externe, se déposent sur la plaine abyssale. Ces glissements sont particulièrement abondants en pied de pente insulaire au Nord-Est et à l'aval de la plate-forme effondrée au Sud-Ouest. Un petit glissement est relevé à l'Ouest au pied de la falaise $F 1$; cette falaise s'inscrit dans le prolongement méridional d'une faille normale pressentie dans la bathymétrie profonde (F2) et dans le lagon (Figure 1b), qui effondre l'édifice volcanique du Nord-Ouest de Mayotte vers l'océan [1]. L'atterrissement du glissement, encore visible en pied de talus, ainsi que la position structurale de cette falaise suggèrent qu'elle corresponde à un escarpement de faille active.

Les glissements sous-marins sont des phénomènes destructifs qui affectent couramment les édifices volcaniques comme la chaîne hawaiienne [18], les Canaries [1], la Polynésie [6] ou La Réunion par exemple [15,21]. Plusieurs mécanismes sont invoqués à l'origine des glissements, dont les intrusions massives de dykes [11, 14, 19], les augmentations des valeurs de pentes en stade post-bouclier [25], les vibrations des séismes [23] et enfin l'imbrication de formations aux caractéristiques géomécaniques médiocres au sein des flancs des volcans [1, 22]. À Mayotte, les formations imbriquées dans les avalanches ne sont pas connues, mais la période la plus propice à ces phénomènes est bien le stade postbouclier avec l'éruption sub-aérienne de formations volcanoclastiques, l'intrusion massive de dykes ou encore l'activité de failles normales décrites plus haut.

Dans les secteurs glissés, l'affaissement progressif des produits d'avalanches puis l'érosion par des courants de turbidité à forte charge sédimentaire va également engendrer des canyons [22]. Ce mécanisme est invoqué à Mayotte pour générer les chenaux manifestement plus récents et de dimensions plus importantes que ceux des zones non déstabilisées. 
Les vastes avalanches de débris peuvent également générer, selon les conditions de mises en place, des séismes (séismes hawaiiens de 1868 et 1975) ou bien des raz-de-marée [18, 19]. À Mayotte, des indices existent en faveur de ces deux types de phénomènes, mais les liens de cause à effet ne sont pas encore établis : on observe par exemple (Figure 3) que les épicentres des séismes de 1936, 1941 (précision faible), 1993 et 2001 (précision bonne) sont situés non loin de glissements ( $S 1$ et S2) ou de failles présumées actives ( $F 1$ et F2). De même, des transcriptions de l'histoire orale de Mayotte font état de raz-de-marée qui auraient détruit des villages du sud de l'île vers le XVIlème siècle [13].

La morphologie concave (S3) observée dans le Sud-Est de Mayotte (Figure 3) est interprétée comme la trace d'un effondrement gravitaire de la totalité du versant (récifbarrière et flanc). Les deux édifices de $400 \mathrm{~m}$ de hauteur observés au centre de la loupe d'arrachement sont probablement d'origine volcanique et pourraient marquer le début de déstabilisation de ce secteur. De tels écroulements catastrophiques liées à l'intrusion du magma dans des flancs pentus sont en effet décrits dans d'autres volcans pour expliquer l'origine de glissements : en domaine aérien, pendant l'éruption spectaculaire du Mont SaintHelens en 1980 [8] ou en domaine sous-marin, à Hawaii [11]. L'implication du récif dans l'effondrement atteste d'un phénomène récent.

Les nombreux reliefs isolés de forme sub-circulaire sont interprétés comme des édifices volcaniques attestant d'une activité magmatique récente sur la pente insulaire. Ces reliefs coniques présentent parfois une forme évasée au sommet, interprétée comme un effondrement du toit de l'intrusion, car la présence de cratères est peu probable à ces profondeurs.

\section{Discussion}

À l'inverse des canyons observés sur toute la pente insulaire, les zones de déstabilisation sont préférentiellement situées au Nord-Est et au Sud-Ouest, alors que les « champs » de reliefs volcaniques sont répartis au Nord-Ouest et au Sud-Est. Cette distribution « croisée » 
ainsi que la morphologie générale de la pente insulaire, permettent de distinguer les principaux ensembles morphostructuraux, et de discuter de l'évolution de l'île en liaison avec les événements magmatiques et tectoniques connus à terre.

Dans le Nord-Est et le Sud-Ouest, la pente est relativement large et caractérisée par l'importance des déstabilisations de flancs. L'ensemble Sud-Ouest représente une vaste structure homogène constituant le principal soubassement de l'île. Son sommet pourrait se situer dans une baie du lagon de Grande-Terre (V4) considérées par certains auteurs [27] comme une ancienne caldeira aérienne d'axe $\mathrm{N} 140^{\circ}$. À terre, cet appareil comporte principalement des laves basaltiques mio-pliocènes et des pitons de phonolites pliocènes [7]. La maturité de son relief à terre, comparée à celle des autres ensembles, en fait l'édifice le plus ancien de Mayotte. Cet appareil correspond au volcan bouclier du sud de Mayotte [7, 27], alors que l'ensemble Nord-Est (V5) correspond au volcan bouclier du nord de Mayotte, dont la mise en place s'achève plus tardivement de 4 Ma à moins de 150000 ans BP [7, 27]. Le secteur Nord-Ouest de l'île (V6) se distingue par une pente insulaire de moindre largeur, la présence de nombreux reliefs volcaniques et une plate-forme effondrée. Ce secteur est limité au Sud par un escarpement orienté $\mathrm{N} 015^{\circ}$, situé dans le prolongement des failles présumées du lagon (Figure 1). L'interruption du récif-barrière dans ce secteur indique un effondrement tectonique local, probablement le long des failles normales présumées du lagon ( $F 1$ et failles parallèles en mer). Cet effondrement, bien que relativement récent (le récif n'a pas encore retrouvé son niveau à la surface de l'eau), n'aurait pas provoqué de déstabilisations catastrophiques comme semble l'indiquer l'absence de zones rugueuses sur la pente insulaire. Cet ensemble V6 formerait un autre complexe volcanique constitutif de Mayotte ; sur le terrain, cet édifice est formé de basaltes et de phonolites mio-pliocènes [7, 27] organisés en reliefs escarpés qui attestent d'un volcanisme plus récent que celui de l'édifice Sud. Mayotte comporterait donc trois appareils volcaniques principaux (V4, V5 et V6) sur lesquels se sont tardivement épanchés des édifices adventifs ( V1, V2 et V3). 
La partie Sud-Est de l'île se distingue à la fois par la présence de très nombreux édifices volcaniques et par le glissement concave (S3) qui interrompt la morphologie générale convexe de la pente insulaire. Sur Petite-Terre, des maars pyroclastiques et des cônes stromboliens quaternaires à sub-actuels ont percé le récif barrière [7, 27, 32]. La partie EstSud-Est de l'île représente donc le domaine le plus actif et le plus récent de Mayotte.

La décompression brutale, marquée par les failles normales au Nord-Ouest et les effondrements au Sud-Est, a probablement participé à la concentration de l'activité éruptive récente dans ces secteurs bien localisés, à terre (dykes etc.) et en mer (essaims d'intrusions), comme cela est observé aux Canaries ou bien sur les flancs du volcan Kilauea à Hawaii $[1,19]$.

\section{Conclusion}

La mise en évidence de nombreux types de structures confirme la complexité de la construction et de l'évolution de Mayotte. L'île comporte trois volcans principaux édifiés entre le Miocène et le Pliocène et sur lesquels se sont épanchés des édifices holocènes à subactuels. À l'instar de nombreuses îles volcaniques, les phénomènes d'érosion et de transfert de matériaux détritiques sur les pentes sont dominants pendant le stade bouclier, ainsi qu'en témoignent les nombreux canyons observés dans tous les secteurs. Le stade post-bouclier semble étroitement contrôlé par la déstabilisation rapide de l'édifice : il est marqué par des glissements de grande ampleur et par la création de zones extensives qui semblent avoir participé à la concentration de l'activité éruptive dans des secteurs bien localisés. II en résulte un modèle où l'activité éruptive de construction de Mayotte s'est concentrée le long d'une direction régionale $\mathrm{N} 140^{\circ}$ (en liaison avec la déformation extensive et/ou gravitaire au Nord-Ouest et au Sud-Ouest), tandis que l'activité de déstabilisation des flancs s'est focalisée de part et d'autre de cette zone éruptive. 


\section{Remerciements}

Ces travaux entrent dans le cadre des activités scientifiques de l'Institut Polaire IPEV en collaboration avec le BRGM, la Direction de l'Agriculture et de la Forêt et la Collectivité Départementale de Mayotte. Nous tenons en particulier à remercier Y. Balut (IPEV) responsable des opérations scientifiques à bord du Marion-Dufresne 2, E. Michel (LCSE) chef de la mission VIGO MD136 à laquelle fut intégrée Bathymay, le SHOM, l'équipe technique de I'IPEV ainsi que l'équipage du N/O Marion-Dufresne 2 pour leur soutien dans cette opération. Nos remerciements vont enfin à B. Field (IGNS), G. Eucher (BRGM) et aux deux rapporteurs anonymes de cet article pour leur commentaires constructifs.

\section{Références}

[1] E. Ancochea, F. Hern, A. Cendrero, J.M. Cantagrel, J.M. Ftister, E. Ibarrola and J. Coello, Constructive and destructive episodes in the building of a young oceanic island, La Palma, Canary Islands, and genesis of the Caldera de Taburiente, Journal of Volcanology and Geothermal Research (1994) 60 (1994) 243-262

[2] J.C. Audru, A. Bitri, J.F. Desprats, C. Mathon, N. Maurillon, P. Sabourault, M. Terrier-Sedan et O. Sedan, Projet risques naturels et érosion à Mayotte : résultats d'année 1, Rapport BRGM (2002) RP51738-FR

[3] J.C. Audru, A. Bitri, J.F. Desprats, C. Mathon, N. Maurillon, J.L. Nédellec, O. Jossot, J.P. Rançon, P. Sabourault, O. Sedan, M. Terrier-Sedan et N. Zornette N. (2004) - Atlas des aléas naturels à Mayotte, Communes de Mamoudzou, Koungou, Dzaoudzi et Pamandzi. Rapport BRGM (2004) RP$53037-F R$

[4] G. Camoin, L. Montaggioni and C. Braithwaite 2004 - Late glacial to post glacial sea levels in the Western Indian Ocean. Marine Geology (2004) 206, p. 119-146

[5] V. Chevillotte, P. Douillet, G. Cabioch, Y. Lafoyd, Y. Lagabrielle et P. Maurizot, Évolution géomorphologique de l'avant-pays du Sud-Ouest de la Nouvelle-Calédonie durant les derniers cycles glaciaires, Comptes Rendus Geoscience (2005), 337, p. 695-701

[6] V. Clouard and A. Bonneville A., A giant landslide on the southern flank of Tahiti Island, French Polynesia, Geophysical Research Letters (2001), 28 n¹1, p. 2253-2256 
[7] D. Debeuf, Étude de l'évolution volcano-structurale et magmatique de Mayotte (archipel des Comores, océan Indien), Thèse de doctorat de l'Université de la Réunion (2004)

[8] F. Donnadieu and O. Merle, Geometrical constraints of the 1980 Mount St. Helens intrusion from analogue models, Geophysical Research Letters (2001), 28, 4, p. 639-642.

[9] F. Dugas, P. Ville et J. Coudray, Étude sismique du lagon sud-ouest de la Nouvelle-Calédonie (Sud-Ouest Pacifique). Paléomorphologies successives et comportement au Quaternaire supérieur du littoral de l'île, Comptes rendus de l'Académie de Sciences (1980), 290, p. 963-966

[10] W.C. Dullo, G.F. Camoin, D. Blomeier, M. Colonna, A. Eisenhauer, G. Faure, J. Casanova and B.A. Thomassin, Morphology and sediments of the fore-slopes of Mayotte, Comoro Islands : direct observations from a submersible, Specialized Publication of the International Association of Sedimentologists (1998) 25, p. 219-236

[11] D. Elsworth and B. Voight, Dyke intrusion as a trigger for large earthquakes and the failure of volcano flanks. Journal of Geophysical Research (1995), 100, p. 6005-6024

[12] C.M. Emerick and R.A. Duncan, Age progressive volcanism in the Comores archipelago, Western Indian Ocean and implications for Somali plate tectonics, Earth and Planetary Sciences Letters (1982), 60, p. 415-428

[13] S. Hachim, Catastrophes! Mayotte perd sa mémoire, Mémoire de DEA de Géographie de I'Université P. Valéry de Montpellier (2004)

[14] A. Le Dez, R.C. Maury, H. Guillou, J. Cotten, S. Blais et G. Guille, L'île de Moorea (Société) : édification rapide d'un volcan bouclier polynésien complexe, Géologie de la France (1998) 3, p. 51-64 [15] J.F. Lénat, P. Bachèlery, A. Bonneville, A. Galdéano, P. Labazuy, D. Rousset et P.M. Vincent, 1990. Structure and morphology of the submarine flank of an active basaltic volcano: Piton de la Fournaise (Réunion Island, Indian Ocean). Océanologica Acta, 10, p. 211-223

[16] J.A. Malod, D. Mougenot, S. Raillard et A. Maillard, Nouvelles contraintes sur la cinématique de Madagascar ; les structures de la chaine Davie. Comptes Rendus de l'Académie des Sciences (1991) 312, p. 1639-1646

[17] O. Merle and A. Borgia, Scaled experiments of volcanic spreading, Journal of Geophysical Research (1996) 101, p.13805-13817

[18] J.G. Moore, D.A. Clague, R.T. Holcomb, P.W. Lipman, W.R. Normark and M.E. Torresan, Prodigious submarine landslides on the Hawaiian Ridge, Journal of Geophysical Research (1989) 94, p. $17465-17484$ 
[19] J.K. Morgan, G.F. Moore, and D.A. Clague, Slope failure and volcanic spreading along the submarine south flank of Kilauea volcano, Hawaii, Journal of Geophysical Research (2003), 108(B9), p. $2415-2439$.

[20] D. Mougenot, J. Hernandez et P. Virlogeux, Structure et volcanisme d'un rift sous-marin : le fossé des Kérimbas (marge nord-Mozambique), Bulletin de la Société Géologique de France (1989) 8, V2, p. $401-410$

[21] J.F. Oehler, P Labazuy and J.F. Lénat, Recurrence of major flank landslides during the last 2 Mahistory of Reunion Island. Bulletin of Volcanology (2004) 66, p. 585-598

[22] J.F. Oehler, B. van Wyk de Vries and P. Labazuy, Landslides and spreading of oceanic hot-spot and arc shield volcanoes on Low Strength Layers (LSLs): an analogue modeling approach. Journal of Volcanology and Geothermal Research (2005)144, p. 169-189

[23] C.H. Okubo, Rock mass strength and slope stability of the Hilina slump, Kilauea volcano, Hawai'i Journal of Volcanology and Geothermal Research (2004), 138, 1-2, p. 43-76

[24] SHOM, lle de Mayotte, cartes de navigation 6237, 6530, 7492, 7493 (1974-2000).

[25] L. Siebert, Large volcanic debris avalanches: characteristics of sources, areas, deposits and associated eruptions. Journal of Volcanology and Geothermal Research (1984), 22, p.163-197.

[26] A. Späth, A. Le Roex et R.A. Duncan, The Geochemistry of lavas from the Comores Archipelago, Western Indian Ocean: Petrogenesis and Mantle Source Region Characteristics, Journal of Petrology (1996), vol. 37-4, p. 961-991.

[27] L. Stieltjes, Carte géologique de Mayotte à 1/50 000 et sa notice. Éd. BRGM, 1988.

[28] M. Terrier, J.C. Audru, M. Bour et P. Dominique (2000) - Étude de l'aléa sismique régional de l'île de Mayotte ; détermination des mouvements sismiques de référence. Rapport BRGM RP-50250-FR.

[29] B.A. Thomassin, A. Arnoux, J. Coudray, C. Froget, B. Gout, G. Kouyoumontzakis, J.P. Masse, Y. Reyre, J.P. Reys et E. Vacelet, La sédimentation actuelle dans le lagon de Mayotte (île volcanique à récif-barrière, SW océan Indien) et son évolution récente en liaison avec les apports terrigènes, Bull. Soc. Géol. France 8 (1989), t. V, n 6, p. 1235-1251.

[30] B.A. Thomassin, Mayotte, île volcanique à large lagon (N. Canal de Mozambique, Océan Indien) : sa construction et celles de ses récifs coralliens. Bulletin des Naturalistes, Historiens et Géographes de Mayotte, $n^{\circ} 1$ (1999), p. 4-18 et 21-30.

[31] R Urgeles, D.G. Masson, M. Canals, A.B.Watts and T.Le Bas (1999) - Recurrent large-scale landsliding on the west flank of La Palma, Canary Islands. J. Geophys. Res. 104, 25331-25348. 
Océanographie / Oceanography

[32] J. Zinke, J.J.G. Reijmer, B.A. Thomassin, W.C. Dullo, P.M. Grootes et H. Erlenkeuser, Postglacial flooding history of Mayotte Lagoon (Comoro Archipelago, southwest Indian Ocean), Marine Geology (2003), 194, p. 181-196.

\section{Figure 1}

(a) Cadre simplifié de la trace supposée du point chaud des Comores, depuis l'atoll des Glorieuses à l'Est jusqu'au volcan actif de Grande-Comore à l'Ouest (Volcan du Karthala à $2263 \mathrm{~m}$ ). Simplified map of the Comoros hot spot volcanic chain, from the Glorioso atoll (250 km to the NE of Mayotte) to the Grande-Comore active volcano (Karthala, $2263 \mathrm{~m}$ ) $250 \mathrm{~km}$ to the NW.

(b) Carte schématique des grands ensembles géologiques et des structures tectoniques supposées de Mayotte, d'après [2, 3, 7 et 27]. Schematic map of main geological formations and structural features of Mayotte island, observed onland and infered within the lagoon, after [2, 3, 7 and 27].

\section{Figure 2 :}

(a) Carte bathymétrique de la pente insulaire de Mayotte (pas du MNT $=50 \mathrm{~m}$ ) avec un estompage depuis le Sud. Multibeam bathymetry map of Mayotte island outerslope. The $D E M$ is made from a 50-m-grid and a relief-shading has been added from the South.

(b) Représentation de la pente insulaire et du relief de Mayotte à la même échelle, avec un point d'observation fictif depuis l'Est vers l'Ouest. Virtual cross-section, showing the Mayotte outerslope and topography at a same scale, as « seen » from the East towards the West.

\section{Figure 3}

Interprétation morphostructurale préliminaire de la carte bathymétrique. Les indices renvoient au texte. Morphostructural preliminary interpretation of the bathymetry map. Letters refer to the text. 


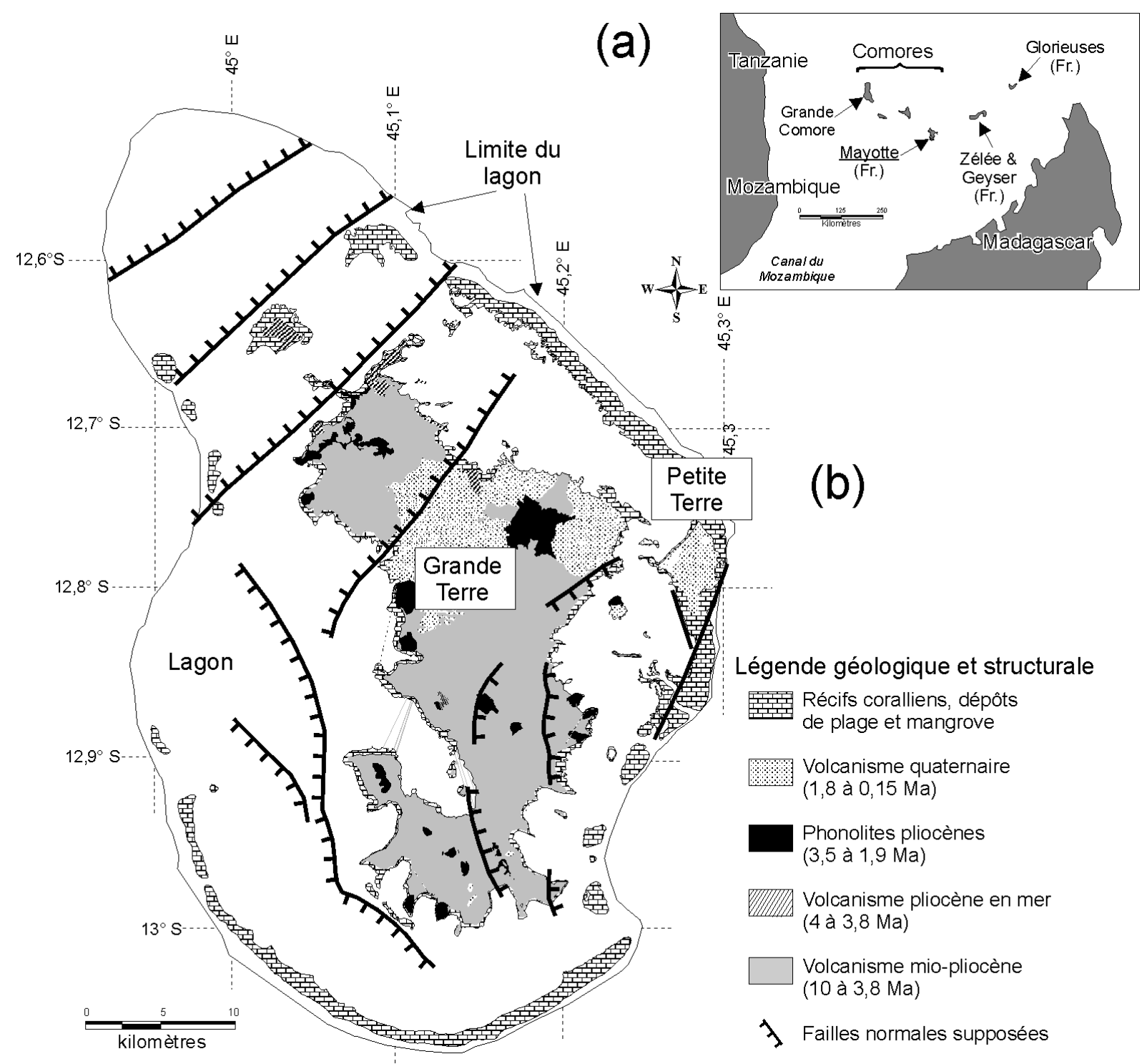

\section{Figure 1}


Océanographie / Oceanography
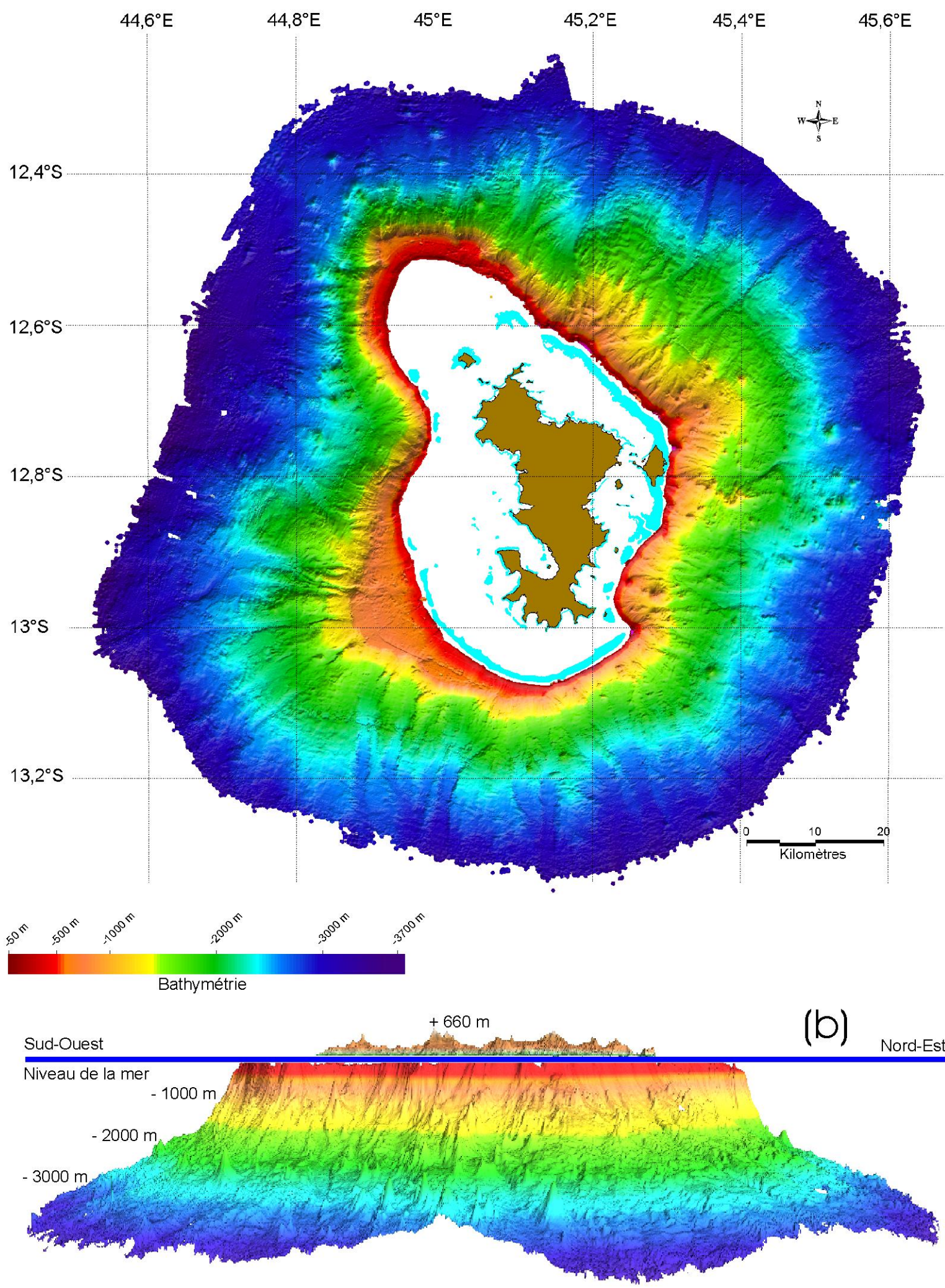

Figure 2 
Océanographie / Oceanography

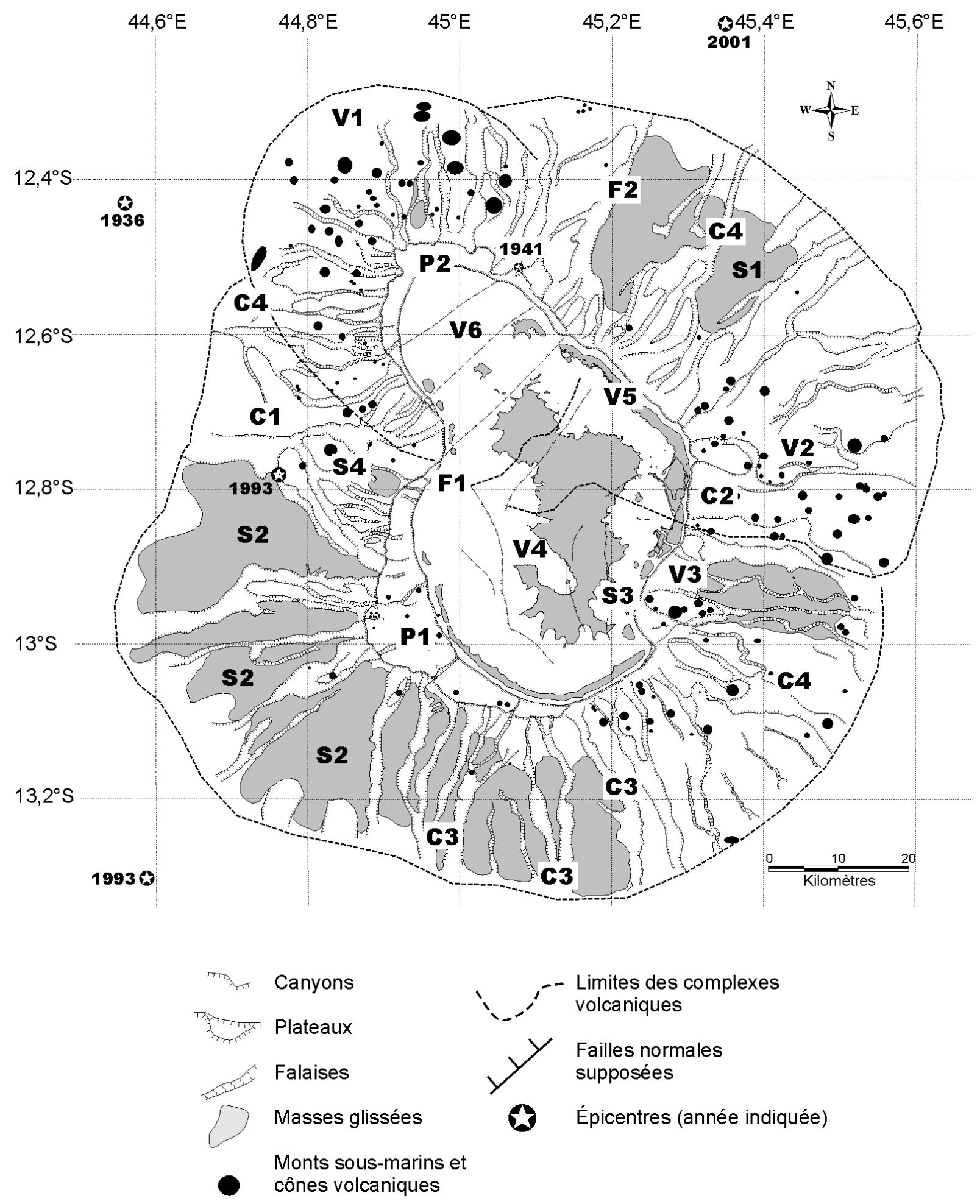

Figure 3 